\title{
LIGHT INTENSITY INFLUENCE ON STRONTIUM TITANATE BASED PHOTO- ELECTROCHEMICAL CELLS
}

\author{
D. HERTKORN*,**, ${ }^{*}$ C. MEGNIN**, C. MÜLLER*, T. HANEMANN**,***, H. REINECKE* \\ *Laboratory for Process Technology, Department of Microsystems Engineering, 79110 Freiburg, Germany \\ **Laboratory for Materials Processing, Department of Microsystems Engineering, 79110 Freiburg, Germany \\ ***Institute for Materials Research, Karlsruhe Institute of Technology, 76133 Karlsruhe, Germany \\ \#E-mail: christof.megnin@imtek.de
}

Submitted January 28, 2017; accepted March 8, 2017

\begin{abstract}
Keywords: Photo-electrochemical cells, $\mathrm{SrTiO}_{3}$, Light intensity, Quantum efficiency
The influence of light intensity on photo-electrochemical cells (PECs) consisting of an n-type strontium titanate ( $\left.\mathrm{SrTiO}_{3}\right)$ photoanode and nickel cathode in potassium hydroxide electrolyte is studied. The band levels of an electrolyte-metalsemiconductor-electrolyte system are presented and the effect of different light intensities on the energy levels is investigated. Photocurrent density, quantum efficiency, and open circuit potential measurements are performed on the processed PECs under different light intensities $(375 \mathrm{~nm})$. It is demonstrated that a threshold value of the light intensity has to be reached in order to obtain positive photo activity and that beyond this value the performance remains nearly constant.
\end{abstract}

\section{INTRODUCTION}

Photo-electrochemical cells (PECs) are an attractive approach for clean hydrogen production. They are based on the splitting of water into hydrogen and oxygen gas under sun light illumination. No additional environmentally harmful by-products are generated, which is a big advantage compared to current hydrogen production methods like steam reforming of methane [1]. There have been investigations on PECs for the last few decades using different approaches and designs with varying cell structures, semiconductor materials, and processing technologies. However, due to numerous requirements in terms of photo-electrochemical and semiconducting properties, finding an optimal material and system is very challenging. Therefore, apart from developing alternative materials with enhanced properties, detailed electrochemical and physical investigations of known materials such as strontium titanate $\left(\mathrm{SrTiO}_{3}\right)$ are also important for a better understanding of the behavior of PECs under working conditions.

In this study we present PECs consisting of an n-type $\mathrm{SrTiO}_{3}$ photoanode and nickel metal cathode in potassium hydroxide electrolyte. $\mathrm{SrTiO}_{3}$ is a perovskitetype semiconductor and possesses excellent stability properties in alkaline media under illumination [2]. Furthermore, it exhibits adequate band positions and a large band gap to split water into hydrogen and oxygen without any additional bias voltage [3]. At first, we discuss the influence of light intensity on the energy levels of a PEC with electrolyte-metal-semiconductor- electrolyte interfaces and then show photocurrent density, external quantum efficiency (EQE) and open circuit potential (OCP) measurements of the presented PECs under different $375 \mathrm{~nm}$ illumination intensities.

\section{THEORETICAL}

When an n-type semiconductor photoanode connected with a metal cathode is introduced into an electrolyte containing a redox couple, a charge transfer from the semiconductor and electrolyte occurs until equilibrium is reached. For such a system this results in a depletion of the majority charge carriers (electrons) and in an upwards bending of the valence and conduction band of the semiconductor. As a consequence, under illumination the photo-generated charge carriers are separated in this electric field. On one side the holes move to the semiconductor-electrolyte interface and perform the oxygen evolution reactions (OER), and on the other side the electrons travel to the metal cathode at the metal-electrolyte interface and react with water, causing the hydrogen evolution reaction (HER). The positions of the energy bands at the semiconductorelectrolyte interface are known as flat band levels of the valence and conduction bands. They have to bracket the redox couple potentials for the OER and HER in order to be able to split water without bias voltage. For metal oxide photoanodes the holes exhibit sufficient potential difference for the OER and are therefore not considered to be a limiting factor. In contrast, the position of the 
conduction band is usually in the spotlight, since its edge is very close to the HER potential. In the case of $\mathrm{SrTiO}_{3}$ it is only about $-200 \mathrm{mV}$ more negative [4]. Figure 1a illustrates that a freely moving electron would not be able to perform the HER, although the semiconductor fulfills the aforementioned requirements.

As described in Equation 1, there are several effects and transitions lowering the electron energy level $E_{e}$. The energy losses from the flat band level $E_{F B}$ include the band bending $E_{B B}$, the energy difference $E_{C F}$ of the conduction band $E_{C B}$ and the Fermi level $E_{F}$, and the required overpotential $\eta_{C B}$ for the HER, leading to an energy level of the electron $E_{e}$ at the metal-electrolyte interface that is too low [5-7]. Note that at the semiconductor-metal interface a charge transfer also occurs, leading to a matching of the work function of the metal and the Fermi level $E_{F}$ of the semiconductor [1].

$$
E_{e}=\left|E_{F B}\right|-\left|E_{B B}\right|-\left|E_{C F}\right|-\left|e \eta_{C B}\right|=E_{f}>E_{\mathrm{HER}}
$$

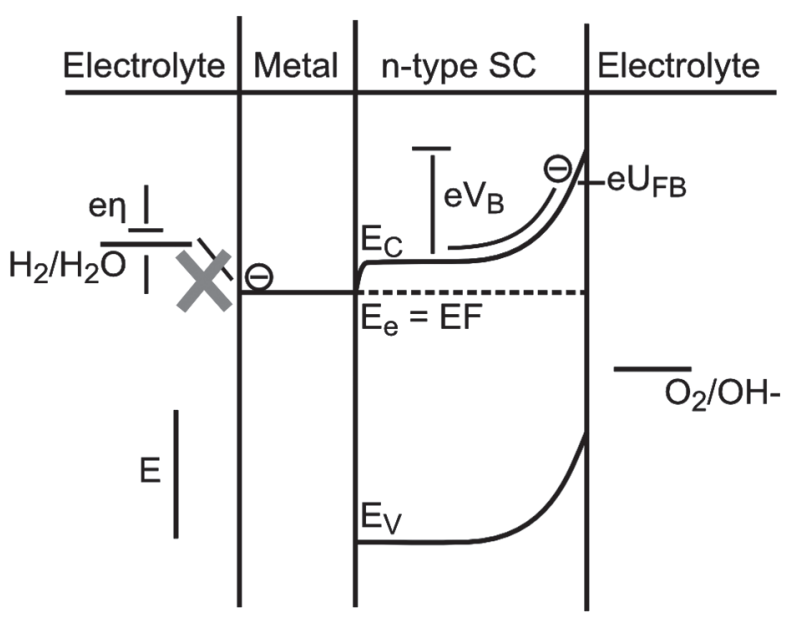

a) without illumination

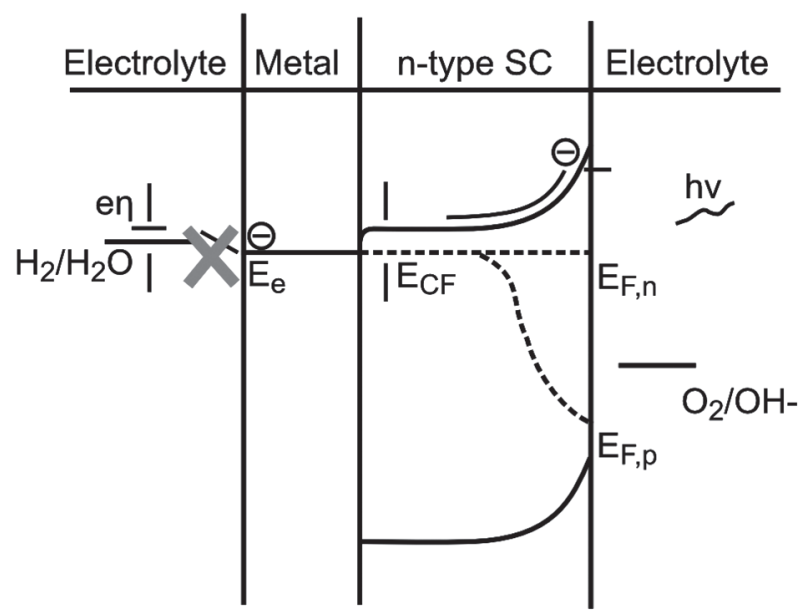

b) with low illumination
Equation 1 reveals that the Fermi level $E_{F}$ has to be higher than the HER energy level $E_{H E R}$ and not just the flat band level $E_{F B}$, which is fixed for a given system [8]. The overpotential $\eta_{C B}$ can be assumed to be fixed, since the density of the light-generated electrons and hence the reaction rates are very low compared to electrolyzers [9]. Therefore, the only way to increase $E_{F}$ is to decrease the band bending $E_{B B}$ and the difference $E_{C F}$ between $E_{C B}$ and $E_{F}$, respectively.

These values change in the presence of photo induced charge carriers as illustrated in Figures $1 \mathrm{~b}$ and 1c. Under steady-state illumination the use of quasi-Fermi levels for both majority $E_{F, n}$ and minority charge carriers $E_{F, p}$ is introduced, since they are no longer identical for electrons and holes. The quasi-Fermi level for electrons $E_{F, n}$ is defined via the Boltzmann constant $k$ and absolute temperature $T$. The values $\Delta \mathrm{n}$ and $n_{0}$ represent the electron concentration created by the incident light and the intrinsic electron concentration, respectively. As shown in Equations 2 and 3, $E_{F n}$ increases with increasing light intensity $I$. However, for an n-type semiconductor $n_{0}$ is very high in comparison to $\Delta \mathrm{n}$, which leads to only a slight shift towards the conduction band [5]. Even for high illumination levels when $\Delta$ n equals $n_{0}$, the $E_{F, n}$ only increases by about $18 \mathrm{meV}$ at room temperature.

$$
E_{F n}=E_{C B}+\left|E_{F C}\right|=E_{F}+k T \ln \left(1+\frac{\Delta n}{n_{0}}\right)
$$

$$
\Delta n \sim I
$$

Band bending is the result of the equilibrium process of the semiconductor electrons and the redox species in the electrolyte. As a consequence the electron concentration at the surface $n_{S}$ is lower than the intrinsic electron concentration $n_{0}$. This means that in the depletion region the additional photo- generated

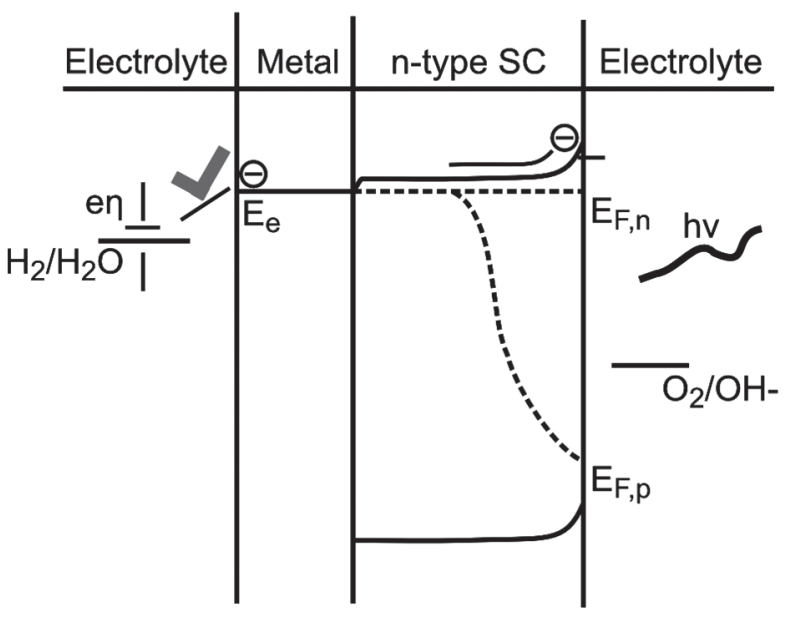

c) with high illumination

Figure 1. Band energy levels for n-type semiconductor-metal PEC in contact with an electrolyte a) without, b) with low, and c) with high illumination; the incident light mainly decreases the band bending EBB leading to a raise of the Fermi level EF, $\mathrm{n}$ of the electrons, so that they possess at a certain intensity level sufficient energy to perform the hydrogen evolution reaction. 
electrons $\Delta \mathrm{n}$ are significant and clearly cause a potential change. The energy loss of the band bending $E_{B B}$, i.e. the energy difference of $E_{F B}$ and $E_{C B}$, can be approximated by a Boltzmann-type relationship [6,7].

$$
E_{B B} \approx k T \ln \left(\frac{n_{s}+\Delta n}{n_{0}}\right) \approx k T \ln \left(1+\frac{\Delta n}{n_{0}}\right)
$$

The surface concentration $n_{S}$ is very small and can be neglected under illumination. Equation 4 shows that $E_{B B}$ changes significantly with the illumination level [10]. Here, when $\Delta \mathrm{n}$ equals $n_{0}$, the band bending vanishes, resulting in a flat band situation. The energy level of the electrons $E_{e}$ increases with high illumination levels, so that they possess sufficient energy to perform the HER. In other words, there has to be a certain light level, where $E_{e}$ equals $E_{H E R}$. This value represents a threshold point, below which the HER is blocked and above which the HER is enabled.

\section{EXPERIMENTAL}

The PECs investigated in this work consist of an n-type $\mathrm{SrTiO}_{3}$ photoanode and a nickel metal cathode. At first, $\mathrm{SrTiO}_{3}$ nano powder (99\%, Advanced Materials) was electrophoretically deposited on nickel substrates (Nickel 201, Tankii Alloy Material) in order to obtain a porous film with a thickness of approximately $5 \mu \mathrm{m}$. Then the samples were sintered in a tube furnace at $1200^{\circ} \mathrm{C}$ for $20 \mathrm{~min}$ with a cooling/heating rate of $10 \mathrm{~K} / \mathrm{s}$ in reducing gas $\left(95 \mathrm{~mol} . \% \mathrm{~N}_{2}+5 \mathrm{~mol} . \% \mathrm{H}_{2}\right.$, Airliquide, Arcal F5) to induce oxygen vacancies in the $\mathrm{SrTiO}_{3}$ lattice and hence create an n-type semiconductor. A detailed description of the process steps is given in [11] The samples have an active area of $10 \times 10 \mathrm{~mm}^{2}$. The electrochemical measurements were performed under different illumination levels in a two-electrode setup to determine the photocurrent and external quantum efficiency (EQE). They were also characterized in a threeelectrode setup to detect the open circuit potential (OCP). The light source (Roithner Laser) has a wavelength of $375 \mathrm{~nm}$, with adjustable intensities ranging from 0.3 to $36.8 \mathrm{~mW} \cdot \mathrm{cm}^{-2}$.

\section{RESULTS}

Figure 2 shows the photocurrent densities and the EQEs under illumination with different intensities. The photocurrent density increases linearly with increasing intensity (light power density) beyond an intensity of $0.8 \mathrm{~mW} \mathrm{~cm}^{-2}$.

This threshold intensity is quite low, but still in the resolution range of the used optical sphere (Coherent PS19Q). Below this value no current is measured. In this case the intensity is too low to sufficiently raise the energy level of the electrons $E_{e}$, and hence the HER cannot proceed. A higher light intensity and hence a higher energy level is needed to allow the generated electrons to react with the electrolyte. Beyond the threshold point the EQE plateau is in the range of $54 \%$ to $60 \%$ and independent of the light intensity. This can be expected, since the photocurrent density depends linearly on the photon flux. The OCP behaves similar to the EQE for different illumination levels.

As illustrated in Figure 3, once the threshold value is reached the redox reactions proceed and the OCP drops steeply. With increasing intensity the OCP further decreases approaching the flat band potential which is approximately $-1.09 \mathrm{~V}$ vs. $\mathrm{Ag} / \mathrm{AgCl}$ [12]. However, although the electrons possess a higher potential at high illumination levels, the EQE remains the same. This also means that the overall efficiency is independent of the light level beyond the threshold point. This is the main difference between solar cells and PECs. The electrons and holes in PECs have to perform the water splitting reaction and therefore need to possess the $E_{H E R}$ and $E_{O E R}$, respectively. This automatically defines the required

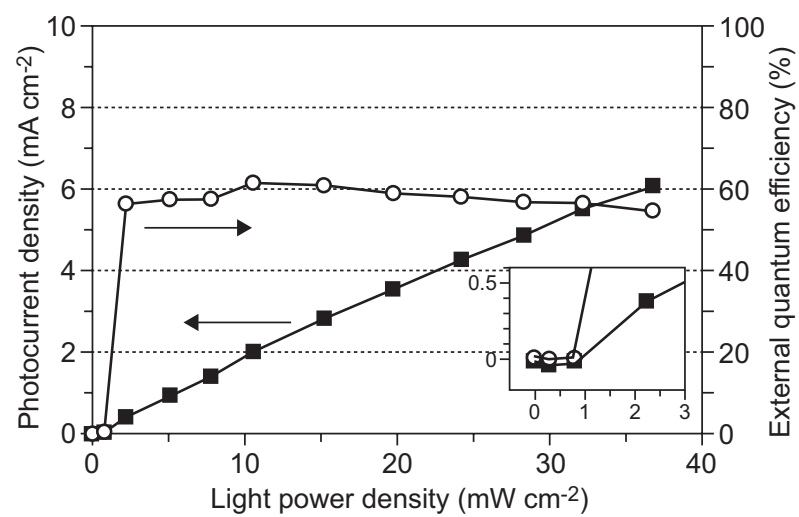

Figure 2. Photocurrent densities and quantum efficiencies under different light powder densities (intensities); a threshold intensity has to be reached to obtain a measurable response.

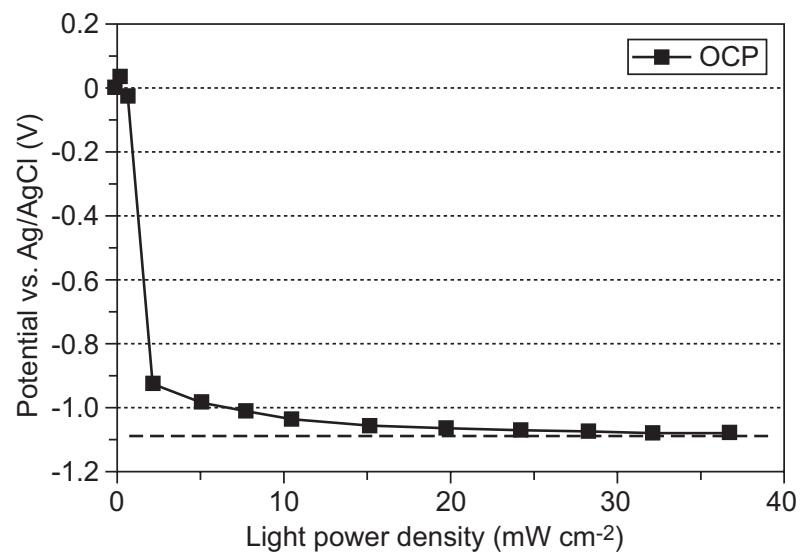

Figure 3. Open circuit potentials under different light power densities; at the threshold intensity the potential drops steeply and further decreases with increasing intensity approaching the flat band potential [12]. 
potential and hence the operation point of the device. In contrast, solar cells are set to work at the optimal operation point. This corresponds to the maximum power point, where the product of the photocurrent and voltage is highest. Thus, the light-induced voltage increase can be exploited in solar cells to get a higher overall efficiency, but not in PECs.

\section{CONCLUSION}

The influence of light intensity on the performance of $n$-type $\mathrm{SrTiO}_{3}$ based photo-electrochemical cells has been investigated. The photo induced charge carriers decrease the band bending of the semiconductor and hence raise the quasi-Fermi level and the energy level of the electrons, respectively. Reaching a certain light intensity enables the hydrogen evolution reaction to be performed. The convergence of the quasi-Fermi level towards the conduction band is small and can be neglected. Photocurrent density measurements reveal that $\mathrm{SrTiO}_{3}$-Ni PECs have a light intensity threshold value that must be met for water splitting to occur. Once this value is reached, the photocurrent density increases linearly with the photon flux and constant quantum efficiencies are exhibited. The open circuit potential drops steeply at the threshold value and further decreases with increasing intensity. However, PECs do not work better with higher intensities beyond the threshold point, since the operation point is defined by the required water splitting potential. In contrast, solar cells are set to work at the maximum power point exploiting the light induced voltage increase.

Acknowledgement

The authors would like to thank the German Research Foundation (Deutsche Forschungsgemeinschaft) for their financial support within the graduate school "Micro Energy Harvesting" (GRK 1322/2).

\section{REFERENCES}

1. Bak T., Nowotny J., Rekas M., and Sorrell C.C. (2002): Photo-electrochemical hydrogen generation from water using solar energy. Materials-related aspects. International Journal of Hydrogen Energy, 27, 991-1022. doi:10.1016/ S0360-3199(02)00022-8

2. Wrighton M.S., Ellis A.B., Wolczanski P.T., Morse D.L., Abrahamson H.B., Ginley D.S. (1976): Strontium Titanate Photoelectrodes. Efficient Photoassisted Electrolysis of Water at Zero Applied Potential. Journal of the American Chemical Society, 98, 2774-2779. doi:10.1021/ja00426a017

3. Mavroides J.G., Kafalas J.A., Kolesar D.F. (2016): Photoelectrolysis of water in cells with $\mathrm{SrTiO}_{3}$ anodes. Applied Physics Letter, 28, 241-243. doi:10.1063/1.88723

4. Maruska H.P., Gosh A.K. (1979): Transition-metal dopants for extending the response of titanate photoelectrolysis anodes. Solar Energy Materials, 1, 237-247. doi:10.1016/ 0165-1633(79)90042-X

5. Tomkiewicz M., Fay H. (1979): Photoelectrolysis of Water with Semiconductors. Applied physics, 18, 1-28. doi:10.1007/BF00935899

6. Gomes W.P., Cardon F. (1982): Electron energy levels in semiconductor electrochemistry. Progress in Surface Science, 12, 155-215. doi:10.1016/0079-6816(82)90002-8

7. Walter M.G., Warren E.L., McKone J.R., Boettcher S.W., Mi Q., Santori E.A., Lewis N.S. (2010): Solar Water Splitting Cells. Chemical Reviews, 110, 6446-6473. doi:10.1021/cr1002326

8. Carp O., Huisman C.L., Reller A. (2004): Photoinduced reactivity of titanium dioxide. Progress in Solid State Chemistry, 32, 33-177. doi:10.1016/j.progsolidstchem. 2004.08.001

9. Suffredini H.B., Cerne J.L., Crnkovic F.C., Machado S.A., Avaca L.A. (2000): Recent developments in electrode materials for water electrolysis. International Journal of Hydrogen Energy, 25, 415-423. doi:10.1016/S0360-3199 (99)00049-X

10. Sanchez C., Sieber K.D., Somorjai G.A. (1988): The photoelectrochemistry of niobium doped $\alpha-\mathrm{Fe}_{2} \mathrm{O}_{3}$. Journal of Electroanalytical Chemistry and Interfacial Electrochemistry, 252, 269-290. doi:10.1016/0022-0728(88) 80216-X

11.Hertkorn D., Elsenheimer H.C., Bruch R., Paul F., Müller C., Hanemann T., Reinecke H. (2013): Thickness variation of electrophoretically deposited strontium titanate films for photoelectrochemical energy conversion. Journal of Applied Physics, 114, 027020. doi:10.1063/1.4811817

12. Chen Z., Jaramillo T.F. Deutsch T., Shwarsctein A., Forman A., Gaillard N., Garland R., Takanabe K., Heske C., Sunkara M., McFarland R., Domen K., Miller E., Turner J., Dinh H. (2010): Accelerating materials development for photoelectrochemical hydrogen production: Standards for methods, definitions, and reporting protocols. Journal of Materials Research, 25, 3-16. doi:10.1557/JMR.2010.0020 